\title{
Parallel Processing of Sensory Input by Bursts and Isolated Spikes
}

\author{
Anne-Marie M. Oswald, ${ }^{1}$ Maurice J. Chacron, ${ }^{1,2}$ Brent Doiron, ${ }^{1,2}$ Joseph Bastian, ${ }^{3}$ and Leonard Maler ${ }^{1}$ \\ ${ }^{1}$ Department of Cellular and Molecular Medicine, University of Ottawa, Ottawa, Ontario K1G 2K4, Canada, ${ }^{2}$ Physics Department, University of Ottawa, \\ Ottawa, Ontario K1N 6N5, Canada, and 3Department of Zoology, University of Oklahoma, Norman, Oklahoma 73019
}

Burst firing is commonly observed in many sensory systems and is proposed to transmit information reliably. Although a number of biophysical burst mechanisms have been identified, the relationship between burst dynamics and information transfer is uncertain. Electrosensory pyramidal cells have a well defined backpropagation-dependent burst mechanism. We used in vivo, in vitro, and modeling approaches to investigate pyramidal cell responses to mimics of behaviorally relevant sensory input. We found that within a given spike train, bursts are biased toward low-frequency events while isolated spikes simultaneously code for the entire frequency range. We also demonstrated that burst dynamics are essential for optimal feature detection but are not required for stimulus estimation. We conclude that burst and spike dynamics can segregate a single spike train into two parallel and complementary streams of information transfer.

Key words: burst; dendritic backpropagation; neural code; stimulus estimation; feature detection; sensory systems

\section{Introduction}

During sensory processing, peripheral input is processed so that relevant aspects of the sensory signal are encoded in the sequences of action potentials that constitute the neural code. In theory, if the stimulus input and the neural output are known, then the transformation between the two defines the coding mechanism. However, because of the complexity of most sensory input and the variability of neural responses, only a few of the mechanisms by which stimuli are represented in neural codes have been identified (Rieke et al., 1997; Eggermont, 1998; Lestienne, 2001).

Action potential sequences can consist of single spikes, highfrequency (HF) spike clusters known as bursts, or both. When coding for comparable signals, bursts, in comparison with single spikes, have been shown to improve the signal-to-noise (SNR) ratio (Sherman, 2001) and transmit specific or additional information about the stimulus (Reinagel et al., 1999; Keat et al., 2001; Kepecs et al., 2002; Martinez-Conde et al., 2002). Bursts also have been suggested to enhance the reliability of information transfer between neurons (Malinow et al., 1994; Lisman, 1997). However, because the behavioral significance of specific stimulus patterns is often unknown; it is difficult to determine whether bursts code for particular categories of relevant stimuli that are distinct from those coded for by single spikes (Krahe and Gabbiani, 2004).

Behaviorally relevant electrosensory inputs have been characterized and easily mimicked (Zupanc and Maler, 1993; MacIver et

Received Feb. 9, 2004; revised March 23, 2004; accepted March 26, 2004.

This work was supported by grants provided by Canadian Institutes of Health Research to L.M., National Institutes of Health to J.B., Natural Sciences and Engineering Research Council of Canada to M.C. and B.D., and Ontario Graduate Scholarship to A.M.O.

Correspondence should be addressed to Anne-Marie Oswald, Department of Cellular and Molecular Medicine, University of Ottawa, 451 Smyth Road, Ottawa, Ontario K1G 2K4, Canada. E-mail: ammoswald@yahoo.com. DOI:10.1523/JNEUROSCI.0459-04.2004

Copyright $\odot 2004$ Society for Neuroscience $\quad$ 0270-6474/04/244351-12\$15.00/0 al., 2001). Specifically, prey stimuli are low-frequency (LF) $(<10$ $\mathrm{Hz}$ ) inputs, whereas communication stimuli are often high frequency $(>40 \mathrm{~Hz})$. The ability of electrosensory pyramidal cells to encode these stimuli depends on both the frequency content and the spatial extent of the stimulus (Bastian et al., 2002; Chacron et al., 2003). In vivo studies of electrosensory pyramidal cell bursting have revealed that spontaneous burst production varies with dendritic structure and spontaneous firing rate (Bastian and Nguyenkim, 2001), and stimulus-evoked bursts extract stimulus features better than single spikes (Gabbiani et al., 1996; Metzner et al., 1998). In addition, pyramidal cells can switch from a nonoscillatory to oscillatory and burst firing pattern contingent on spatial stimulus characteristics (Doiron et al., 2003a). However, the mechanism underlying burst production was not addressed in these studies.

In vitro studies have established that the biophysical burst mechanism of electrosensory pyramidal cells is dependent on the active dendritic backpropagation of somatic spikes (Turner et al., 1994; Lemon and Turner, 2000), and theoretical analyses also have characterized these burst dynamics (Doiron et al., 2001, 2002; Laing and Longtin, 2003; Laing et al., 2003). These previous studies suggest that the electrosensory system is an ideal preparation in which to elucidate the role of a biophysically and dynamically characterized burst mechanism in sensory coding.

This study addresses bursting and stimulus encoding in the weakly electric fish, Apteronotus leptorhynchus. Using both in vivo and in vitro experiments and an accurate burst model (ghostburster), we demonstrated that bursts exclusively code for lowfrequency stimuli, whereas isolated spikes code for highfrequency stimuli. We also demonstrated that optimal burst coding is dependent on burst dynamics. Thus, the electrosensory system creates two "streams" of frequency-specific information that could be differentially decoded by higher brain centers. 


\section{Materials and Methods}

In vivo. The preparation and surgical techniques for recording from Apteronotus leptorhynchus have been described previously (Bastian, 1996a,b) and were in accordance with the University of Oklahoma animal care and use guidelines. Stimulation consisted of random amplitude modulations (RAMs) of the animal's own electric organ discharge (EOD) produced by adding an amplitude-modulated train of sinusoidal signals (EOD mimic) to the animal's normal discharge. The RAMs were produced by multiplying the sinusoids with band-limited Gaussian noise (0-60 Hz bandwidth; eighth order Butterworth filter) and applied with local geometry (Bastian et al., 2002) using a small dipole (2 $\mathrm{mm}$ tip spacing) at a distance of 2-3 mm lateral to the skin surface. Extracellular and intracellular recordings of 33 pyramidal cells were obtained primarily from the centrolateral (CLS) and lateral (LS) electrosensory lateral line lobe (ELL) segments. Extracellular single-unit recordings were made with metal-filled electrodes, and intracellular recordings were made with 40-100 M , KCl-filled micropipettes. Recordings were preamplified and acquired with Cambridge Electronics Design (Cambridge, UK) 1401plus hardware and Spike II software.

In vitro. Transverse brain slices from the ELL of A. leptorhynchus were prepared in accordance with animal care protocols of the University of Ottawa as described previously (Berman et al., 1997). The slices were maintained at room temperature $\left(20-22^{\circ} \mathrm{C}\right)$ in oxygenated artificial CSF (ACSF) for 1-2 hr before recording. The ACSF contained (in mM): 124 $\mathrm{NaCl}, 3 \mathrm{KCl}, 0.75 \mathrm{KH}_{2} \mathrm{PO}_{4}, 2 \mathrm{CaCl}_{2}, 2 \mathrm{MgSO}_{4}, 24 \mathrm{NaHCO}_{3}, 10$ D-Glucose. All chemicals were provided by Sigma (St. Louis, MO).

The pyramidal cell layer is located just below the stratum fibrosum (StF), an easily identifiable landmark in the ELL slice (Berman et al., 1997). Intracellular recordings from 37 ELL pyramidal cells from the entire pyramidal cell layer were made using $80-120 \mathrm{M} \Omega$ boroscillate glass electrodes, pulled by a Brown-Flaming P-87 puller (Sutter Instruments, Novato, CA), and filled with 2 м KAc. Of these cells, 24 were identified as bursting neurons by direct current injection and analyzed.

Recordings were obtained primarily from centromedial segment (CMS). Differences in oscillatory and burst activity across the ELL segments have been reported both in vitro (Turner et al., 1996) and in vivo (Metzner et al., 1998). Consequently, a comparison of 16 CMS, 6 CLS, and 2 LS in vitro pyramidal cell responses was conducted. The cells showed no significant differences in any parameters (such as firing rate, membrane potential, and coherence) studied, including bursting, and consequently the data were pooled.

After impalement, the neurons were allowed to stabilize for $\sim 5-10$ min. If necessary, a constant $0.1-0.5 \mathrm{nA}$ hyperpolarizing current was injected through the recording electrode to stabilize the cells. A series of depolarizing current steps was injected to determine the current at which the firing rate was $\sim 10-20 \mathrm{~Hz}(0.1-0.5 \mathrm{nA})$, which is within the range of spontaneous activity in vivo.

Previous studies have used Gaussian noise stimuli to stimulate the receptive fields of pyramidal cells in vivo (Bastian et al., 2002; Chacron et al., 2003, Doiron et al., 2003a). The membrane potential of pyramidal cells can follow these fluctuations (Chacron et al., 2003) presumably because electroreceptor EPSPs are brief (Berman and Maler, 1998). We therefore injected similar noise signals via the recording electrode into pyramidal cells in vitro. The noise stimuli were Gaussian noise with cutoff frequencies of $0-60,0-20$, or $40-60 \mathrm{~Hz}$ and SD of $0.5 \mathrm{nA}$, unless otherwise indicated. This was added to a $100 \mathrm{sec}$ depolarizing current $(0.1-0.5$ $\mathrm{nA}$, as determined above). Stimulation was a computer-controlled (IgorPro and Pulse Control; WaveMetrics, Lake Oswego, OR) (Herrington et al., 1995), and pyramidal cell responses were amplified (Axoclamp 2A; Axon Instruments, Burlingame, CA), sampled at $10 \mathrm{KHz}$, and stored for analysis (IgorPro and Pulse Control).

Drug application. Tetrodotoxin (TTX; $15 \mu \mathrm{M}$ in ACSF; Sigma) was focally pressure ejected in the ventral molecular layer above the Stf in a region corresponding to the apical dendrites of the pyramidal cells (Turner et al., 1994).

The model neuron. The previously described two-compartment model of an ELL pyramidal cell known as the ghostburster model (Doiron et al., 2002) contains all the essential elements to reproduce bursting as detailed by Lemon and Turner (2000) and Doiron et al. (2001). The model neuron is comprised of an isopotential soma (s) and a single dendritic compartment that are joined through and axial resistance of $1 / \mathrm{gm}_{\mathcal{c}}$, allowing for the electrotonic diffusion of currents from the soma to dendrite $(\mathrm{d})$ and vice versa. Both compartments contain the essential spiking currents; fast inward $\mathrm{Na}^{+}\left(I_{\mathrm{Na}, \mathrm{s}}, I_{\mathrm{Na}, \mathrm{d}}\right)$ and outward delayed rectifying (Dr) $\mathrm{K}^{+}\left(I_{\mathrm{Dr}, \mathrm{s}}, I_{\mathrm{Dr}, \mathrm{d}}\right)$, and passive leak currents $\left(I_{\text {leak }}\right)$. The presence of spiking currents in the dendrite enables the active backpropagation of somatic action potentials required for bursting. The membrane potentials at the soma, $V_{\mathrm{s}}$, and the dendrite, $V_{\mathrm{d}}$, are determined using a Hodgkin-Huxleylike formalism (Koch, 1999). The original ghostburster model (Doiron et al., 2002) comprised six nonlinear differential equations; this study expands on this model to incorporate persistent sodium currents $\left(I_{\mathrm{NaP}, s}\right.$, $\left.I_{\mathrm{NaP}, \mathrm{d}}\right)$ and a slower activation of spiking sodium in the dendrite. The input current $I_{s}(t)$ delivered to the soma was comparable with the zeromean Gaussian noise $(0-60 \mathrm{~Hz})$ that was added to constant depolarizing current $I_{0}$ and injected in vitro. $I_{s}(t)$ was produced by a fourth-order Butterworth filter, and the $\mathrm{SD}\left(\sigma=3.7 \mu \mathrm{A} / \mathrm{cm}^{2}\right)$ of $I_{\mathrm{s}}(t)$ and the intensity of the offset current $I_{0}\left(0.21 \mu \mathrm{A} / \mathrm{cm}^{2}\right)$ were adapted so that the firing rate $(32 \mathrm{~Hz})$ and burst fraction $(0.24)$ were similar to the in vitro recording seen in Figure 1. $V_{\mathrm{s}}$ and $V_{\mathrm{d}}$ are described by the following equations:

$$
\begin{aligned}
C_{\mathrm{m}} \frac{d V_{\mathrm{s}}}{d t} & =I_{0}+I_{\mathrm{s}}(t)+g_{\mathrm{Na}, \mathrm{s}} \cdot m_{\infty}^{2} \cdot\left(1-n_{\mathrm{s}}\right) \cdot\left(V_{\mathrm{Na}}-V_{\mathrm{s}}\right) \\
& +g_{\mathrm{NaP}, \mathrm{s}} \cdot m_{\infty, \mathrm{NaP}, \mathrm{s}}^{2} \cdot\left(V_{\mathrm{Na}}-V_{\mathrm{s}}\right) \\
& +g_{\mathrm{Dr}, \mathrm{s}} \cdot n_{\mathrm{s}}^{2} \cdot\left(V_{\mathrm{K}}-V_{\mathrm{S}}\right)+\frac{g_{\mathrm{c}}}{\kappa} \cdot\left(V_{\mathrm{d}}-V_{\mathrm{s}}\right)+g_{\text {leak }} \cdot\left(V_{1}-V_{\mathrm{s}}\right) \\
C_{\mathrm{m}} \frac{d V_{\mathrm{d}}}{d t} & =g_{\mathrm{Na}, \mathrm{d}} \cdot m_{\mathrm{d}}^{2} \cdot h_{\mathrm{d}} \cdot\left(V_{\mathrm{Na}}-V_{\mathrm{d}}\right)+g_{\mathrm{NaP}, \mathrm{d}} \cdot m_{\infty, \mathrm{NaP}, \mathrm{d}}^{2} \cdot\left(V_{\mathrm{Na}}-V_{\mathrm{d}}\right) \\
& +g_{\mathrm{Dr}, \mathrm{d}} \cdot n_{\mathrm{d}}^{2} \cdot P_{\mathrm{d}} \cdot\left(V_{\mathrm{K}}-V_{\mathrm{d}}\right)+\frac{g_{\mathrm{c}}}{1-\kappa} \cdot\left(V_{\mathrm{s}}-V_{\mathrm{d}}\right) \\
& +g_{\text {leak }} \cdot\left(V_{\mathrm{l}}-V_{\mathrm{d}}\right) .
\end{aligned}
$$

The parameter $g$ is a maximal conductance $\left(g_{\max }, \mathrm{mS} / \mathrm{cm}^{2}\right)$, whereas $m$ and $s$ are activation parameters, and $h, n$, and $p$ are inactivation parameters. As described previously (Doiron et al., 2002), the activation of spiking $\mathrm{Na}^{+}$in the soma, $m_{\infty, s}$, instantaneously tracks the membrane voltage, $V_{\mathrm{s}}$, and its inactivation, $h_{\mathrm{s}}$, is modeled as $h_{\mathrm{s}} \approx 1-n_{\mathrm{s}}$, where $n_{\mathrm{s}}$ is the activation parameter for $I_{\mathrm{Dr}, \mathrm{s}}$ described by:

$$
\frac{d n_{\mathrm{s}}}{d t}=\frac{n_{\infty}\left(V_{\mathrm{s}}\right)-n_{\mathrm{s}}}{\tau_{\mathrm{n}, \mathrm{s}}} .
$$

To simplify the ghostburster model, the activation of dendritic spiking $\mathrm{Na}^{+}$was originally slaved to the dendritic voltage as described for $I_{\mathrm{Na}, \mathrm{S}}$. This resulted in burst inter-spike intervals (ISIs) that were $<3 \mathrm{msec}$, which were rarely seen experimentally. Immunocytochemical and electrophysiological data suggest that spiking $\mathrm{Na}^{+}$channels are confined to active zones along the proximal apical dendrite. This type of channel distribution can lead to delays in depolarizing after potential (DAP) production that are not accounted for in the ghostburster, resulting from the single compartment dendrite. Thus, we describe the activation of spiking $\mathrm{Na}^{+}$in the dendrite, $m_{\mathrm{d}}$, by:

$$
\frac{d m_{\mathrm{d}}}{d t}=\frac{m_{\infty, \mathrm{d}}\left(V_{\mathrm{d}}\right)-m_{\mathrm{d}}}{\tau_{\mathrm{m}, \mathrm{d}}} .
$$

This resulted in doublet ISIs that were $4-6 \mathrm{msec}$ and closer to experimental values ( $5-8 \mathrm{msec}$ ).

The inactivation of spiking $\mathrm{Na}^{+} h_{\mathrm{d}}$ in the dendrite, as well as the 
activation $n_{\mathrm{d}}$ and the slow inactivation $p_{\mathrm{d}}$ of the dendritic $\mathrm{K}^{+}$currents, are modeled as described by Doiron et al. (2002):

$$
\begin{gathered}
\frac{d h_{\mathrm{d}}}{d t}=\frac{h_{\infty, \mathrm{d}}\left(V_{\mathrm{d}}\right)-h_{\mathrm{d}}}{\tau_{\mathrm{h}, \mathrm{d}}}, \\
\frac{d n_{\mathrm{d}}}{d t}=\frac{n_{\infty, \mathrm{d}}\left(V_{\mathrm{d}}\right)-n_{\mathrm{d}}}{\tau_{\mathrm{n}, \mathrm{d}}}, \\
\frac{d p_{\mathrm{d}}}{d t}=\frac{p_{\infty, \mathrm{d}}\left(V_{\mathrm{d}}\right)-p_{\mathrm{d}}}{\tau_{\mathrm{p}, \mathrm{d}}} .
\end{gathered}
$$

Persistent sodium $(\mathrm{NaP})$ currents may modulate bursting (Doiron et al., 2003b). Thus, for completeness and a better fit to experimental data, we incorporate $\mathrm{NaP}$ currents in both the soma and the dendrite as described by Doiron et al. (2001). This increased the excitability of model neuron and lowered bias current required to quantitatively reproduce in vitro results. For simplicity, we slave the activation of $\mathrm{NaP}, m_{\mathrm{NaP}}$, to the membrane voltage in both the soma and the dendrite.

The voltage dependence of each activation and inactivation parameter is described by steady state conductance curves as presented for the activation of somatic spiking $\mathrm{Na}^{+}, m_{\infty}$ :

$$
m_{\infty}\left(V_{\mathrm{S}}\right)=\frac{1}{1+e^{-\left(V_{\mathrm{s}}-V_{1 / 2}\right) / k}} .
$$

The values for $g_{\max }, \tau, V_{1 / 2}$, and $k$ for each current are given in Table 1 . Other parameter values are as follows: the ratio of somatic to total area, $\kappa=0.6$; the reversal potentials, $V_{\mathrm{Na}}=40 \mathrm{mV}, V_{\mathrm{K}}=-88.5 \mathrm{mV}, V_{\text {leak }}=$ $-70 \mathrm{mV}$; membrane capacitance, $C_{\mathrm{m}}=1 \mu \mathrm{F} / \mathrm{cm}^{2}, g_{\text {leak }}=0.22 \mathrm{mS} / \mathrm{cm}^{2}$, $g_{\mathrm{c}}=0.21 \mathrm{mS} / \mathrm{cm}^{2}$. In some simulations, $g_{\mathrm{Na}, \mathrm{d}}$ was set to zero to remove active dendritic spiking and prevent bursting. This does not affect $\mathrm{NaP}$ conductance.

Equations 1-7 were integrated in time via a fourth-order Runge-Kutta approximation scheme with a time step of $2.5 \mu \mathrm{sec}$. All simulations were $200 \mathrm{sec}$ of simulation time.

Data analysis. In vivo, in vitro, and model results were analyzed using IgorPro and MATLAB (Mathworks, Natick, MA). The first second of the spike train was not analyzed to exclude transients resulting from the onset of the stimulus. We determined ISI criteria to identify burst ISIs on the basis of the valleys of a bimodal ISI histogram $(10 \mathrm{msec}$; in vitro, model neuron) and a previously used criterion for deviations from Poisson statistics (Bastian and Nguyenkim, 2001) (12 $\pm 4 \mathrm{msec}$; in vivo). Generally, ISIs $<10 \mathrm{msec}$ are in agreement with backpropagationdependent burst ISIs reported in vitro (Noonan et al., 2003). Using the ISI criteria, the spike trains were separated into three different spike trains for analysis. The first, denoted "all spikes," was the raw spike train that contained the timing of all spikes. The second contained the timing of only the first spike of the bursts, denoted "burst events." The final was denoted "isolated spikes" and contained only the timing of spikes that were not burst spikes. The coherence $C(f)$ between the three individual spike trains and the stimulus was calculated according to $C(f)=$

\begin{tabular}{|c|c|c|c|c|}
\hline Current & $g_{\max }\left(\mathrm{mS} / \mathrm{cm}^{2}\right)$ & $V_{1 / 2}(\mathrm{mV})$ & $k(\mathrm{mV})$ & $\tau$ (msec) \\
\hline$I_{\mathrm{Na}, \mathrm{s}}\left[m_{\infty, s}\left(V_{s}\right)\right]$ & 55 & -40 & 3 & NA \\
\hline$I_{\text {NaP, }, S}\left[m_{, \infty, s}\left(V_{S}\right)\right]$ & 0.14 & -58.5 & 6 & NA \\
\hline$I_{D r, s}\left[n_{s}\left(V_{s}\right)\right]$ & 20 & -40 & 3 & 0.39 \\
\hline$I_{\text {Nad }}\left[m_{d}\left(V_{d}\right) / h r_{d}\left(V_{d}\right)\right]$ & 3.1 & $-40 /-52$ & $5 /-5$ & $0.3 / 4.0$ \\
\hline$I_{\text {NaP,d }}\left[m_{\infty, \mathrm{d}}\left(V_{\mathrm{d}}\right)\right]$ & 0.1 & -58.5 & 6 & NA \\
\hline$I_{\text {Drd }}\left[n_{d}\left(V_{d}\right) / p_{d}\left(V_{d}\right)\right]$ & 35 & $-40 /-65$ & $5 /-6$ & $6 / 15$ \\
\hline
\end{tabular}
$\left|P_{\mathrm{sx}}(f)\right|^{2} /\left[P_{\mathrm{ss}}(f) P_{\mathrm{xx}}(f)\right]$, where $P_{\mathrm{ss}}$ and $P_{\mathrm{xx}}$ are the power $(\mathrm{P})$ spectrums of the stimulus and the spike train, respectively, and $P_{\mathrm{sx}}$ is the cross-

Table 1. Model parameter values

Both somatic (s) and dendritic (d) compartments contain the essential spiking currents; fast inward $\mathrm{Na}^{+}\left(I_{\mathrm{Na}, s} I_{\mathrm{Na}, \mathrm{d}}\right)$ and outward delayed rectifying (Dr) $\mathrm{K}^{+}\left(I_{\mathrm{Dr}, \mathrm{s}} I_{\mathrm{Dr}, \mathrm{d}}\right)$ as well as persistent $\mathrm{Na}^{+}\left(I_{\mathrm{NaP},{ }^{\prime}} I_{\mathrm{NaP}, \mathrm{d}}\right)$. The parameters for the steady state conductance curves for each current are the maximum conductance $\left(g_{\max }\right)$, half-deactivation voltage $\left(V_{1 / 2}\right)$, deactivation time constant $(\tau)$, and the deactivation slope $(k)$. In the case of two entries under $V_{1 / 2}, \tau$, and $k$, the first value corresponds to the activation parameter and the second value to the deactivation parameter. NA, Not applicable. spectrum between the stimulus and the spike train. Coherence takes a value between 0 and 1 and is a measure of the strength of the neural response to a stimulus at a given stimulus frequency $(f)$.

Burst- and spike-triggered averages. We determined $400 \mathrm{msec}$ regions of the stimulus centered on each isolated spike or burst event for the in vivo and in vitro spike trains to be the spike-triggered average (STA) and burst-triggered average (BTA), respectively. To assess the specificity of spikes or bursts, we reinjected $0.5 \mathrm{~Hz}$ trains of STAs or BTAs with an offset current of $+0.1 \mathrm{nA}$ into a small number of pyramidal cells in vitro.

Feature detection. We used the feature extraction technique described in detail by Metzner et al. (1998). Briefly, the spike train and stimuli were binned at $\Delta t=0.5 \mathrm{msec}$, so that no more than one spike occurred per bin. For each bin, the stimulus vector was determined as the stimulus waveform preceded the bin $(t-\Delta t ; t)$. These vectors were then grouped as distributions according to whether a spike occurred, $P\left(s / \lambda=1_{\text {all }}\right)$, a spike occurred and it was an isolated spike, $P\left(s / \lambda=1_{\text {isol }}\right)$, a spike occurred and it was the first spike of a burst, $P\left(s / \lambda=1_{\text {burst }}\right)$, or a spike did not occur (null), $P(s / \lambda=0)$. The means $\left(m_{\mathrm{i}}\right)$, which are the spike, isolated spike, burst-, and null-triggered averages, respectively, as well as the covariances $\left(\Sigma_{\mathrm{i}}\right)$ of the distributions, were estimated. Fisher's linear discriminant function was used to determine optimal feature vector $(f)$ to maximize the SNR.

$$
\begin{gathered}
\frac{1}{2}\left(\Sigma_{0}+\Sigma_{1}\right) \cdot f=m_{1}-m_{0}, \\
\text { SNR }=\frac{\left[f^{\mathrm{T}} \cdot\left(m_{1}-m_{0}\right)\right]^{2}}{f^{\mathrm{T}} \cdot\left(\frac{1}{2} \Sigma_{0}+\frac{1}{2} \Sigma_{1}\right) \cdot f} .
\end{gathered}
$$

The probability of correct detection, $P_{\mathrm{D}}$ (correctly classifying a stimulus vector as eliciting or not eliciting a spike), and the probability of false alarm, $P_{\mathrm{FA}}$ (misclassifying a stimulus vector as eliciting or not eliciting a spike), can then be determined. The separations between each of the distributions $P\left(f^{\mathrm{T}} \cdot s / \lambda=1_{\text {all }}\right), P\left(f^{\mathrm{T}} \cdot s / \lambda=1_{\text {burst }}\right), P\left(f^{\mathrm{T}} \cdot s / \lambda=1_{\text {isol }}\right)$, and null distribution $P\left(f^{\mathrm{T}} \cdot s / \lambda=0\right)$ were assessed using these techniques, and the receiver operating characteristic (ROC) curve $\left(P_{\mathrm{D}}\right.$ vs $\left.P_{\mathrm{FA}}\right)$ was plotted for each case.

Statistics. The statistical significances of the results were assessed using paired $t$ tests.

\section{Results}

\section{Pyramidal cell burst responses to noise stimuli applied in vivo and in vitro}

The stimulus consisted of broadband Gaussian noise $(0-60 \mathrm{~Hz})$ presented as AMs of electrosensory input in vivo and intracellular current injection in vitro. The mean firing rates in response to the stimulus were similar in vitro $(18 \pm 4 \mathrm{~Hz})$ and in vivo $(22 \pm 13$ $\mathrm{Hz}$ ), and the spike trains were qualitatively similar in that they consisted of isolated spikes as well as high-frequency clusters of two to four spikes (Fig. $1 A 1, B 1$, arrowheads). The majority of ISI histograms both in vivo and in vitro had initial sharp peaks at the shortest intervals (Fig. 1A2,B2) and could not be fit by a single exponential $\left(\chi^{2}>500\right)$; thus the spike train statistics are nonPoissonian, suggestive of burst discharge.

Based on the ISI histograms (Fig. 1 A2,B2), an ISI criterion was established to identify spikes belonging to bursts (Turner et al., 1994, 1996; Debusk et al., 1997; Metzner et al., 1998; Reinagel et al., 1999). A criterion ISI of $10 \mathrm{msec}$ was chosen for in vitro data. Because in vivo pyramidal cells show a wide range of firing frequencies, criterion ISIs were individually chosen for each cell (see Materials and Methods). The mean ISI criterion in vivo was $12 \pm$ $4 \mathrm{msec}$. The burst fraction, defined as the total number of spikes contained within bursts divided by the total number of spikes, was determined for each cell, and the means were $0.49 \pm 0.16$ in 


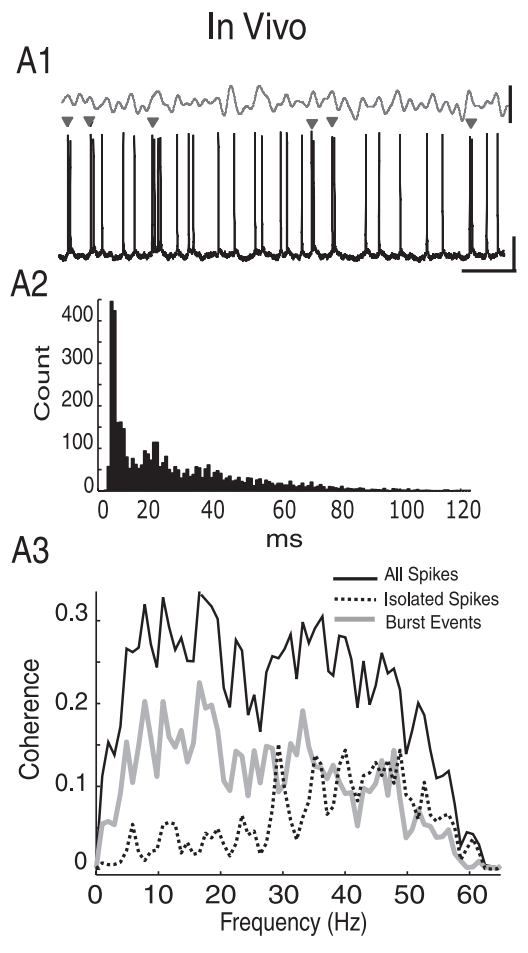

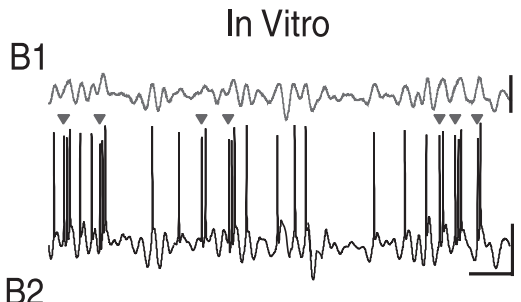

B2
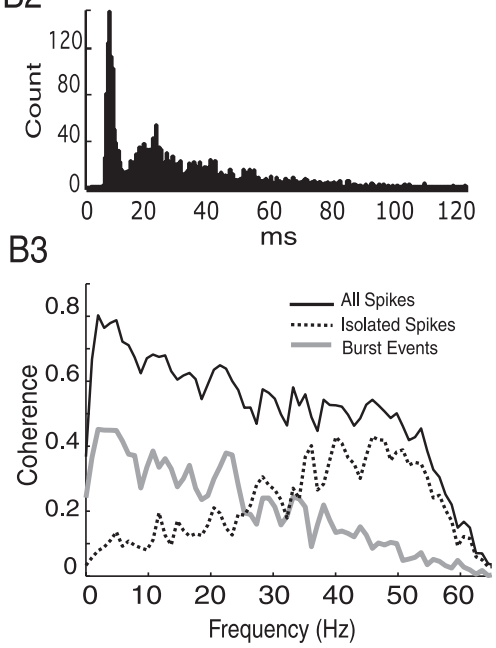

Figure 1. ELL pyramidal cell responses to broadband $(0-60 \mathrm{~Hz})$ Gaussian noise stimuli. $A$, A pyramidal cell response in vivo to a localized application of the broadband stimulus at the skin (firing rate, $40 \mathrm{~Hz}$; burst fraction, 0.49 ). B, A pyramidal response in vitro to direct current injection of the broadband stimulus (firing rate, $33 \mathrm{~Hz}$; burst fraction, 0.27 ). $A 1, B 1$, Spike trains in response to broadband stimuli (gray) consisted of isolated spikes and high-frequency bursts of spikes (arrowheads). A2, B2, ISI histograms with a significant peak in the 3-10 msec range that corresponds to the high-frequency spike events. $A 3, B 3$, Coherence plots between the stimulus and the neural response for the full spike train (black solid line) and the partitioned spike trains: burst events (gray line) and isolated spikes (dashed line). Calibration: (in A) stimulus, $0.18 \mathrm{mV} / \mathrm{cm}$; spike train, $20 \mathrm{mV} ; 100 \mathrm{msec}$. Calibration: (in B) stimulus, $0.5 \mathrm{nA}$; spike train, $20 \mathrm{mV} ; 100 \mathrm{msec}$.

vivo and $0.22 \pm 0.12$ in vitro. The burst criterion was then used to separate the full spike train into two component trains: bursts, which consisted of the timing of the first spike in a burst event, regardless of the number of spikes in a burst, and isolated spikes, which consisted of the timing of the remaining single spikes.
The coherence between each of the event trains (all spikes, burst events, and isolated spikes) and the stimulus was calculated (Fig. 1A3,B3). The mean coherence values between 0 and $20 \mathrm{~Hz}\left(C_{\text {low }}\right)$ and 40 and $60 \mathrm{~Hz}\left(C_{\text {high }}\right)$ were calculated to assess the frequency selectivity of the event trains. These and other measured values are reported in Table 2, and the results of statistical analysis are presented in the text. Previous in vivo studies (Chacron et al., 2003) have demonstrated that pyramidal cells give low-pass responses on stimulation of their receptive field center, and this was confirmed in the sample reported here $\left(C_{\text {low }}=0.24 \pm 0.12 ; C_{\text {high }}=0.10 \pm 0.097\right.$; $p<0.01)$. Analysis of the in vitro full spike trains corroborated that the pyramidal cells were also low pass (in vitro, $C_{\text {low }}=$ $0.47 \pm 0.081 ; C_{\mathrm{high}}=0.308 \pm 0.08 ; p<$ $0.01)$. The low-pass response can be appreciated in the ratios of $C_{\text {low }}: C_{\text {high }}$, which were 2.4 and 1.5, in vivo and in vitro, respectively.

\section{Bursts and isolated spikes code for different signal components}

Pyramidal cells were capable of responding to the entire stimulus frequency range with bursts or isolated spikes. However, both in vivo and in vitro, burst responses were significantly biased toward low-frequency stimulus events $\left(C_{\text {low }}>C_{\text {high }}\right.$; in vivo and in vitro, $p<0.01$ ) because the $C_{\text {low }}: C_{\text {high }}$ ratio in vivo and in vitro was 4.7 and 3 , respectively. In contrast, isolated spikes are on average less selective $\left(C_{\text {low }} \approx C_{\text {high }}\right.$ : in vivo, $p>0.5$; in vitro, $\left.p>0.5\right)$ with $C_{\text {low: }}$ : $C_{\text {high }}$ ratios of $0.7-1.0$. We also investigated the frequency selectivity of bursts by injecting low-frequency $(0-20 \mathrm{~Hz})$ or high-

Table 2. Summary of measured values in vivo, in vitro, and the model

\begin{tabular}{|c|c|c|c|c|c|c|c|c|}
\hline \multirow{3}{*}{$\begin{array}{l}\text { Stimulus } \\
\text { Condition }\end{array}$} & \multirow{3}{*}{$\begin{array}{l}\text { In Vivo } \\
0-60 \mathrm{~Hz}\end{array}$} & \multicolumn{5}{|l|}{ In Vitro } & \multirow{2}{*}{\multicolumn{2}{|c|}{$\begin{array}{l}\text { Model } \\
0-60 \mathrm{~Hz}\end{array}$}} \\
\hline & & $0-60 \mathrm{~Hz}$ & $0-20 \mathrm{~Hz}$ & $40-60 \mathrm{~Hz}$ & \multicolumn{2}{|c|}{$0-60 \mathrm{~Hz}$} & & \\
\hline & & & & & Control & TTX & ADM & PDM \\
\hline Firing rate (spikes/sec) & $22 \pm 12.9$ & $18 \pm 4$ & $16 \pm 3$ & $16 \pm 2$ & 18 & 18 & $32 \mathrm{~Hz}$ & $33 \mathrm{~Hz}$ \\
\hline Burst fraction & $0.49 \pm 0.16$ & $0.22 \pm 0.12$ & $0.32 \pm 0.19$ & $0.008 \pm 0.005$ & 0.16 & 0.08 & 0.24 & 0.064 \\
\hline$n$ & 33 & 24 & 6 & 6 & 3 & 3 & NA & NA \\
\hline \multicolumn{9}{|l|}{ All spikes } \\
\hline$C_{\text {low }}$ & $0.24 \pm 0.12$ & $0.47 \pm 0.08$ & $0.51 \pm 0.09$ & NA & 0.5 & 0.5 & 0.67 & 0.72 \\
\hline$C_{\text {high }}$ & $0.10 \pm 0.10$ & $0.31 \pm 0.08$ & NA & $0.60 \pm 0.10$ & 0.32 & 0.34 & 0.38 & 0.39 \\
\hline \multicolumn{9}{|l|}{ Burst events } \\
\hline$C_{\text {low }}$ & $0.13 \pm 0.08$ & $0.13 \pm 0.07$ & $0.15 \pm 0.07$ & NA & 0.19 & 0.12 & 0.27 & 0.14 \\
\hline$C_{\text {high }}$ & $0.03 \pm 0.03$ & $0.04 \pm 0.02$ & NA & $0.02 \pm 0.01$ & 0.05 & 0.03 & 0.16 & 0.07 \\
\hline \multicolumn{9}{|l|}{ Isolated spikes } \\
\hline low & $0.08 \pm 0.04$ & $0.28 \pm 0.08$ & $0.42 \pm 0.05$ & $\begin{array}{l}\text { NA } \\
054+013\end{array}$ & 0.25 & 0.33 & 0.19 & 0.47 \\
\hline \multicolumn{9}{|l|}{ BTA $^{-\operatorname{lngn}}$} \\
\hline$P_{\text {low }}$ & $0.041 \pm 0.055$ & $0.20 \pm 0.06$ & & & & & & \\
\hline$P_{\text {high }}$ & $0.009 \pm 0.054$ & $0.05 \pm 0.01$ & & & & & & \\
\hline \multicolumn{9}{|l|}{ STA ${ }^{\text {nign }}$} \\
\hline$P_{\text {low }}$ & $0.021 \pm 0.054$ & $0.06 \pm 0.03$ & & & & & & \\
\hline$P_{\text {high }}$ & $0.010 \pm 0.016$ & $0.04 \pm 0.01$ & & & & & & \\
\hline
\end{tabular}

Stimuli were Gaussian noise ranging from 0 to 60,0 to 20 , and 40 to $60 \mathrm{~Hz}$ and were applied in vivo, in vitro, or in the model as indicated. The mean firing rate and burst fraction during stimulation are reported. In some cases, the stimulus was applied in the presence or absence of TTX in vitro or in the presence of active dendritic backpropagation (ADM) or the absence of backpropagation (PDM). Coherence between the stimuli and the full spike trains, burst trains, and isolated spike trains were measured. $C_{\text {low }}$ and $C_{\text {high }}$ correspond to the mean values of the coherence measured in the $0-20$ and $40-60 \mathrm{~Hz}$ ranges, respectively. The power spectrums of the BTA and STA were obtained. $P_{\text {low }}$ and $P_{\text {high }}$ correspond to the mean power measured in the $0-20$ and $40-60 \mathrm{~Hz}$ ranges, respectively. NA, Not applicable. 

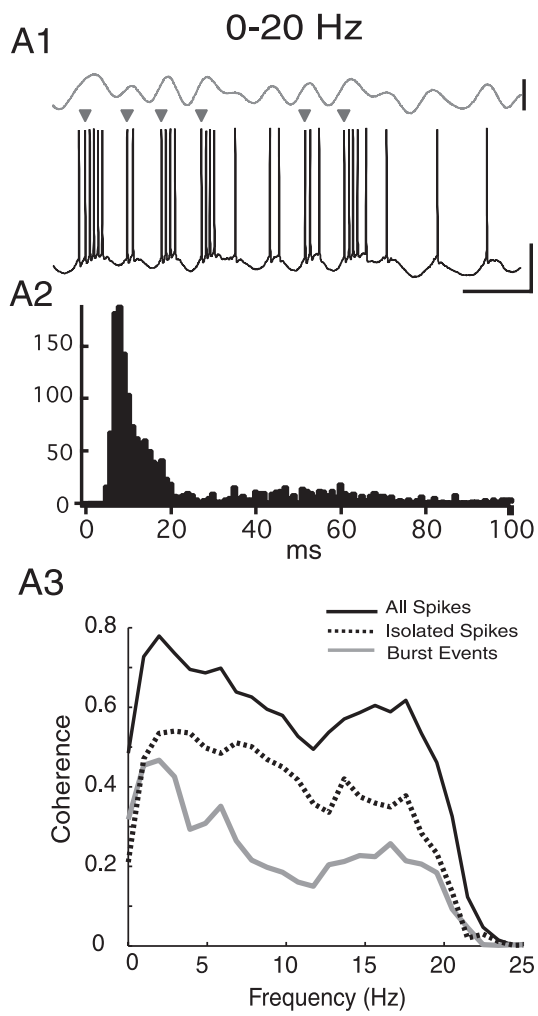

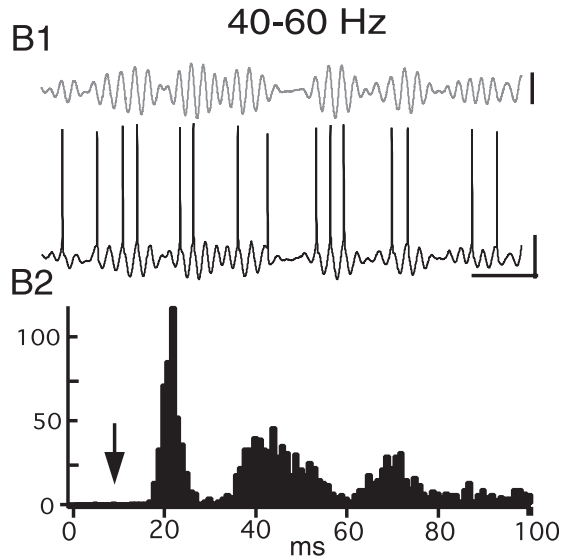

B3

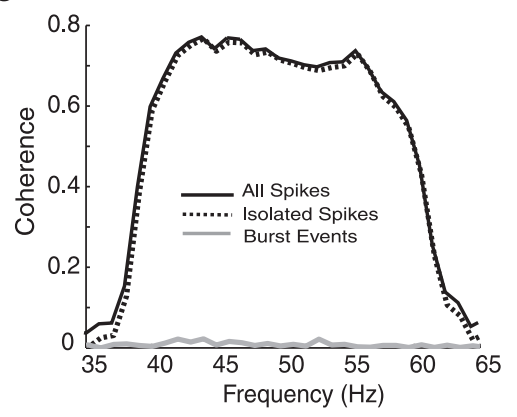

Figure 2. Pyramidal cell responses in vitro to low-frequency $(0-20 \mathrm{~Hz})$ versus high-frequency $(40-60 \mathrm{~Hz})$ Gaussian noise stimuli. $A 1$, The pyramidal cell response (black) to direct current injection of a $0-20 \mathrm{~Hz}$ noise stimulus (gray) consisted of single spikes and high-frequency bursts of two to eight spikes (arrowheads). Firing rate, $20 \mathrm{~Hz}$; burst fraction, 0.49 . A2, ISI histogram is strongly skewed toward short 3-10 msec ISIs. A3, Coherence between the low-frequency stimulus and the neural response for the full spike train (black solid line) and the partitioned spike trains: burst events (gray line) and isolated spikes (dashed line). B1, The pyramidal cell response (black) to direct current injection of a $40-60 \mathrm{~Hz}$ noise stimulus (gray) consisted primarily of single spikes. Firing rate, $20 \mathrm{~Hz}$; burst fraction, 0.00 . B2, The ISI histogram is multimodal but lacks short 3-10 msec ISIs (arrow). B3, Coherence between the high-frequency stimulus and the neural response for the full spike train (black solid line) and the partitioned spike trains: burst events (gray line) and isolated spikes (dashed line). Calibration: stimulus, $0.5 \mathrm{nA}$; spike train, $20 \mathrm{mV}$; $100 \mathrm{msec}$.

frequency $(40-60 \mathrm{~Hz})$ noise in vitro (Fig. $2 A, B$, respectively). During LF stimulation, pyramidal cells responded with both bursts ( $2 \pm 1$ bursts/sec; burst fraction, $0.32 \pm 0.19$ ) and isolated spikes (10 \pm 3 spikes/sec), whereas HF stimulation yielded almost exclusively isolated spikes $(16 \pm 2 \mathrm{~Hz}$; burst fraction, $0.008 \pm 0.005)$. These differential responses are apparent in the ISI histograms where LF stimulation resulted in bursts (Fig. $2 \mathrm{Al}$, arrowheads) consisting of two to eight spikes corresponding to a peak at 3-10 msec (Fig. 2A2) that were absent during HF stimulation (Fig. 2 B2, arrow). During LF stimulation, both bursts and isolated spikes were coherent with stimulus, although isolated spikes had higher coherence values (bursts: $C_{\text {low }}, 0.15 \pm 0.07$; isolated spikes: $\left.C_{\text {low }}, 0.42 \pm 0.05\right)$ (Fig. $2 A 3$ ). In addition, isolated spikes were coherent with the HF stimulus $\left(C_{\text {high }}, 0.54 \pm 0.13\right)$ (Fig. 2B3). These results show that the isolated spikes can be coherent with either LF or HF stimuli and are therefore capable of stimulus estimation over the entire frequency range. Although bursts are selectively elicited by LF stimuli, the lower burst rate results in lower coherence values than those of isolated spikes. Thus, even at low frequencies, isolated spikes outperform bursts for stimulus estimation.

To characterize the signals that evoke bursts versus single spikes, we computed the BTA and STA (Fig. 3A). Both in vivo and in vitro, the power spectra of the BTA and STA are qualitatively similar to the coherence curves for burst events and isolated spikes, respectively (Fig. 3B). The BTA has power predominantly at low frequencies $\left(P_{\text {low }}>P_{\text {high }}\right.$; in vivo, $p=6.8 \times 10^{-4}$; in vitro, $p=1 \times$ $10^{-9}$ ), whereas the STA has significant power over the entire frequency range $\left(P_{\text {low }} \approx P_{\text {high }}\right.$; in vivo, $p>0.1$; in vitro, $p>0.1)$.

We then explored the dependence of bursts on the amplitude and temporal characteristics of the BTA in vitro. As expected, reinjection of BTA and STA waveforms resulted in bursts $(81 \pm 8.5 \%$ of presentations) and spikes (94 $\pm 2 \%)$, respectively (Fig. 3C). The BTA was of significantly greater amplitude $(p<0.01)$ and half-width $(p<0.01)$ than the STA. In some systems, the BTA and STA are scaled versions of each other (Gabbiani et al., 1996; Reinagel et al., 1999), suggesting that increasing the stimulus amplitude would be sufficient to induce bursting. However, scaling the STA to the height of the BTA produced bursts in only $10 \pm 3 \%$ of stimulus presentations. Alternatively, we doubled the SD of the $0-60 \mathrm{~Hz}$ stimulus. The spectral properties of the BTA and STA were conserved (data not shown), but the amplitude of the STA was increased to $0.6 \mathrm{nA}$, a value that surpasses the amplitude of the BTA under control conditions $(0.45 \mathrm{nA})$. This very strong stimulus increased low-frequency burst coherence but had a minimal effect on burst coherence with high frequencies $\left(\Delta C_{\text {high }}\right.$, $0.037 \pm 0.002 ; \Delta C_{\text {low }}>\Delta C_{\text {high }} ; p<0.01$; $n=14)$. These results demonstrate that bursting is dependent on the temporal characteristics of the stimulus as well as its amplitude; this conclusion is consistent with theoretical studies of electrosensory pyramidal cell burst dynamics (Laing and Longtin, 2003).

Bursts in ELL pyramidal cells therefore appear to selectively signal low-frequency components of sensory input, whereas isolated spikes are able to encode the entire frequency range. The bursts analyzed above were empirically defined from the ISI histogram, and we next explore their relationship to the dynamically defined ghostburst mechanism.

\section{Backpropagation-dependent bursting}

ELL pyramidal cells have a well characterized burst mechanism that is dependent on the active backpropagation of somatic spikes along the proximal apical dendrite (Lemon and Turner, 2000; Doiron et al., 2001, 2002). We used constant current injections (0.5-1.0 nA) in vitro to verify that backpropagation-dependent bursting can be elicited in all neurons studied. Figure 4 shows dendritic $(A)$ and somatic $(B)$ recordings in vitro in response to a constant depolarizing current injection. Somatic spikes backpropagate along the dendrite and broaden as the burst evolves. This is reflected at the soma as an increasing DAP (Fig. $4 B$, inset). Finally, DAP potentiation reaches the threshold for highfrequency doublet generation (Fig. $4 B$, arrow). However, the 


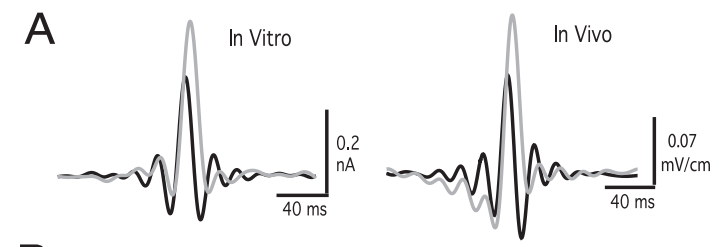

$\mathrm{B}$
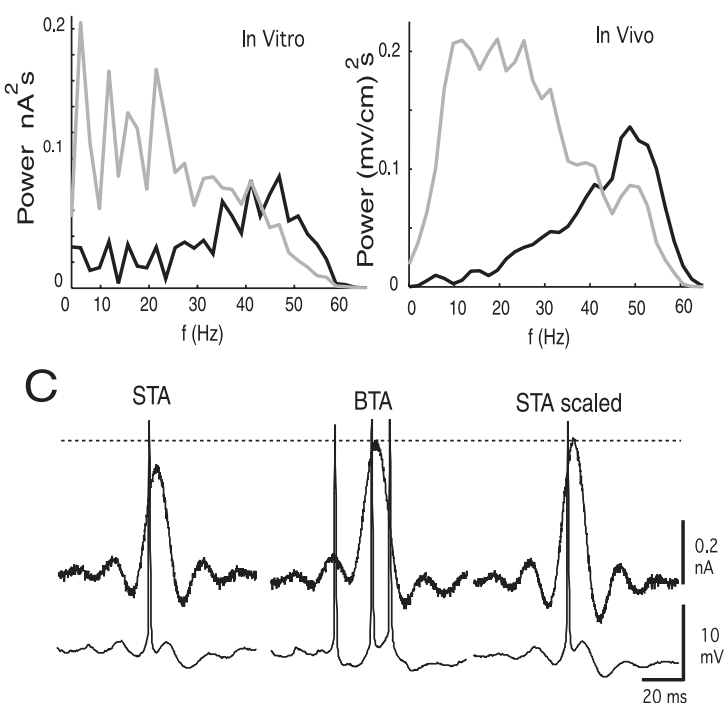

Figure 3. Burst- and spike-triggered averages are distinct stimulus features. A, BTA (gray) and STA (black) in vitro (left) and in vivo (right) were of significantly different height and duration (generated on the basis of the responses to $0-60 \mathrm{~Hz}$ stimulation of the cells presented in Fig. 1). B, The power spectrum of the BTA (gray) was significantly low pass, and that of the STA (black) was broadband to high pass both in vitro and in vivo. C, Reinjection of the STA or BTA in vitro produced single spikes and doublets, respectively. Scaling the STA to the amplitude of the BTA did not elicit doublets in the majority of presentations.

doublet ISI falls within the dendritic spike refractory period halting the backpropagation of the second action potential of the doublet (Fig. $4 A$, asterisk), consequently terminating the burst. The doublet ISIs constitute a substantial peak in the $3-10 \mathrm{msec}$ range of the ISI histogram (Turner et al., 1994; Doiron et al., $2003 \mathrm{~b}$ ). In addition, $0-60 \mathrm{~Hz}$ stimulation in vitro yields ISI histograms with a prominent peak in the $3-10 \mathrm{msec}$ range (Fig. 1 B2) and results in dendritic failures (Fig. 4D). Likewise, ISI histograms with a peak in a similar time range are observed in response to $0-60 \mathrm{~Hz}$ stimulation of a subset of pyramidal cells with high spontaneous firing frequencies (Fig. 1A2). Bursting and dendritic failure can also be elicited by step increases in EOD amplitude in some pyramidal cells in vivo (Fig. 4C). Thus, we propose that the high-frequency doublets present during $0-60 \mathrm{~Hz}$ stimulation are attributable to the backpropagation-dependent burst mechanism in vitro. This same mechanism is likely to underlie bursting in at least a subset of cells in vivo; however, additional studies are needed to determine whether the same mechanism gives rise to bursting in pyramidal cells with lower spontaneous firing frequencies (Bastian and Nguyenkim, 2001).

It has been demonstrated that the backpropagation of somatic action potentials along the dendrite is dependent on voltagedependent sodium $\left(\mathrm{Na}^{+}\right)$conductances (Turner et al., 1994; Doiron et al., 2003b). We focally applied TTX to the proximal apical dendrite of pyramidal cells in vitro. As demonstrated previously (Turner et al., 1994) this eliminated bursting induced by constant current (data not shown). TTX application also greatly reduced the number of high-frequency doublets elicited during

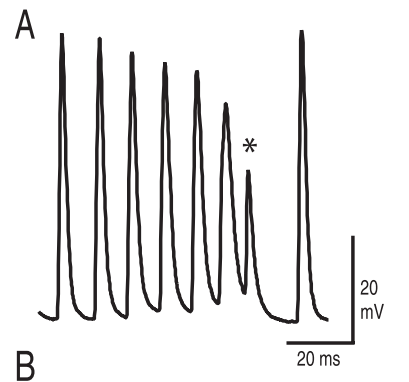

C

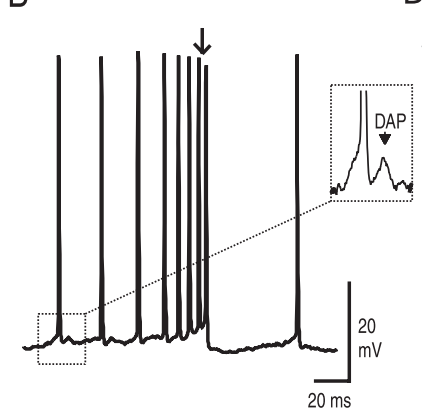

D

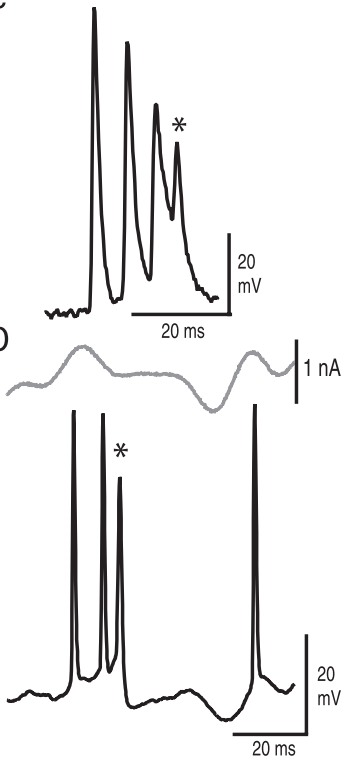

Figure 4. Evidence for backpropagation-dependent bursting in vitro and in vivo. $A$, Dendritic recording of a failure $\left(^{*}\right)$ of spike backpropagation elicited by step depolarization in vitro. $B$, Somatic recording of a backpropagation-dependent burst elicited by step current in vitro. Note the DAP resulting from a dendritic spike (inset) that leads to high-frequency doublet generation (arrow). C, Dendritic failure in response to a step change in the EOD amplitude in vivo. D, Dendritic failure in response to broadband noise stimulation (gray) in vitro.

$0-60 \mathrm{~Hz}$ noise injection (Fig. $5 A$ ), and the ISI histogram became unimodal (Fig. $5 B$ ); note that the average firing rate did not change significantly. These results show that the bursts seen during broadband in vitro current injection are attributable to active dendritic processes.

Because bursts are low-frequency coherent, we hypothesized that preventing bursts during broadband current injection would decrease the low-frequency coherence of the full spike train. During TTX application, there was an expected reduction in burst coherence that coincided with an increase in low-frequency coherence for isolated spikes (Fig. 5C,D). However, surprisingly, there was not a substantial change in full spike train coherence (Fig. $5 C, D)(n=3)$. Contrary to our hypothesis, these results suggest that only the first spike of a burst is important for lowfrequency coherence, and that bursts do not appear to code additional information about the stimulus.

\section{Single spikes effectively code low-frequency stimuli}

TTX applied to the apical dendrites eventually diffuses to the soma and blocks spiking; thus, there was only a limited time for data acquisition during which recordings were stationary and the somatic $\mathrm{Na}^{+}$was not affected. Therefore, we also investigated the role of backpropagation-dependent bursts in stimulus encoding using a two-compartment reduction of the detailed ionic model of an ELL pyramidal cell (Doiron et al., 2001, 2002). The model parameters and stimulation protocols (see Materials and Methods) were adapted so that the firing rate and burst fractions reported for the model were comparable with those presented in vitro (Fig. $1 B$ ).

Injection of $0-60 \mathrm{~Hz}$ Gaussian noise into the model neuron yielded similar results to those seen in vivo and in vitro. Spike trains at the soma contained both single spikes and bursts (Fig. $6 A 1$, arrowheads). The majority of bursts were high-frequency doublets, which corresponded to failures in the dendrite (Fig. 

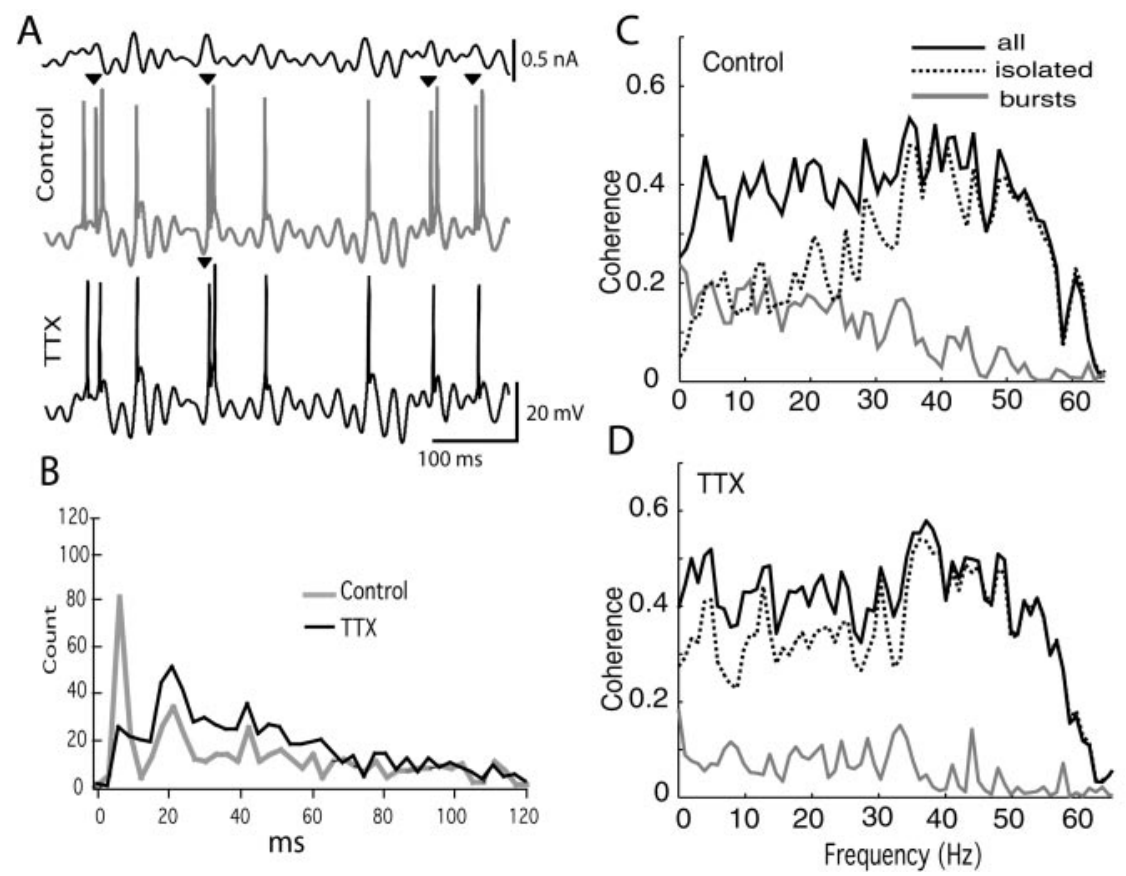

Figure 5. TTX blocks bursting but does not affect overall low-frequency coherence. A, Pyramidal cell response to a broadband stimulus (top trace) before (gray) and after (black) application of TTX to the apical dendrite. The number of bursts (arrowsheads) is greatly reduced by TTX, which is reflected in the decreased peak in the 3-10 msec range of the ISI histogram ( $B)$, control (gray), and TTX (black). C, Coherence of bursts (gray), isolated spikes (black dashed line), and all spikes (black solid line) with the stimulus before application of TTX. D, In the presence of TTX, burst coherence decreased (gray), whereas isolated spike coherence (black dashed line) increased. The coherence of the full spike train did not change significantly (black solid line).

$6 A 1$, asterisks). The ISI histogram was bimodal with peaks at 3-5 and $20 \mathrm{msec}$ (Fig. $6 \mathrm{~B}$, thin gray line). An ISI criterion of $6 \mathrm{msec}$, which corresponds to the trough of the ISI histogram, was chosen to distinguish burst spikes. Based on this criterion, the coherence between each of the event trains (all spikes, burst events, and isolated spikes) and the stimulus were calculated as described above. Similar to pyramidal cells in vivo and in vitro, the model neuron was low pass, and bursts were low-frequency coherent, whereas single spikes had a broadband response (Table 2; Fig. 6C, compare with Fig. 1).

We mimicked TTX blockade of active dendritic processes and prevented burst discharge by setting the dendritic, spiking $\mathrm{Na}^{+}$ conductance $\left(g_{\max , \mathrm{Na}, \mathrm{d}}\right)$ to zero, effectively making the dendrite passive. From this point on, this will be referred to as the passive dendrite model (PDM), whereas the model with active dendritic processes intact will be referred to as the active dendrite model (ADM). The injection of a $0-60 \mathrm{~Hz}$ stimulus into the PDM results in an ISI histogram that still contains high-frequency ISIs $(8-10 \mathrm{msec})$, but the bimodality and the highest frequency doublet ISIs ( $<6 \mathrm{msec}$ ) are lost (Fig. 6A2,B, thick black line). As with experimental blockade, there was a large reduction in burst fraction and low-frequency burst coherence (Table 2) (data not shown). Because burst events are reduced to single spikes, there is a substantial increase in the low-frequency coherence of isolated spikes (Fig. 6D, dashed lines). Consistent with experimental data, there was no substantial change in the coherence of the full spike train (Fig. $6 D$, solid lines). Thus, removing active backpropagation may result in a loss or delay of the second spike of the doublet, but this appears to be inconsequential, because the majority of information appears to be coded in the first spike of a burst.

\section{Bursts and isolated spikes implement different coding strategies}

Although single spikes provide better estimates of the detailed time course of the stimulus, it has been suggested that bursts may be more reliable indicators of specific stimulus features (Lisman, 1997). We used the Fisher linear discriminant (Gabbiani et al., 1996; Metzner et al., 1998) to evaluate the effectiveness of feature detection by burst events versus single spikes. Burst events were distinguished from single spikes on the basis of the previously described ISI criteria, and the following events were identified: all spikes (bursts plus isolated spikes), burst events, isolated spikes, and the null distribution (no bursts or isolated spikes). Stimulus features that corresponded to the $50 \mathrm{msec}$ stimulus epoch that preceded each event were identified and then grouped into distributions according to the event elicited. An optimal feature was determined for each event distribution that maximized the separation between that event distribution and the null distribution. Each individual stimulus feature was then compared with the optimal feature and, on the basis of the degree of similarity, a prediction was made as to whether or not that event (single spike, burst, or null) would occur. The probability of correctly predicting an event versus the probability of false alarm is plotted as the ROC curve.

Figure 7 shows the feature extraction of bursts versus single spikes in response to $0-60 \mathrm{~Hz}$ stimulation in representative pyramidal cells in vivo (Fig. $7 A$ ) and in vitro (Fig. $7 B$ ) as well as to $0-20$ and $40-60 \mathrm{~Hz}$ stimulation in vitro (Fig. $7 C, D$ ). The optimal features (solid lines) detected by all spikes (black), bursts (thick gray), and isolated spikes (dashed) in response to each stimulus are presented in Figure $7 A 2, B 2, C 2$, and D2. The distributions arising from the projection of the stimulus features onto the optimal features are shown in Figure 7A3, B3, C3, and D3; a reduced overlap between the event distributions (bursts, gray; isolated spikes, dashed), and the null distribution (black line) indicates better feature detection.

In response to $0-60 \mathrm{~Hz}$ stimulation, both in vivo and in vitro, bursts have higher SNR values (Fig. 7A1,B1, insets) than the full $\left(\mathrm{SNR}_{\mathrm{B}}>\mathrm{SNR}_{\mathrm{F}}, p<0.01\right)$ and isolated $\left(\mathrm{SNR}_{\mathrm{B}}>\mathrm{SNR}_{\mathrm{I}}, p<0.01\right)$ spike trains. In addition, isolated spikes have lower $\mathrm{SNR}$ values than the full spike train $\left(\mathrm{SNR}_{\mathrm{F}}>\mathrm{SNR}_{\mathrm{I}}, p<0.01\right)$. The SNRs in vitro are much higher than in vivo because of the much lower rate of spontaneous activity in vitro compared with in vivo. Comparable results to in vitro responses were also obtained for the same stimulus in the model neuron (data not shown). The optimal features are derived from the BTA (low pass) and STA (broadband), which differ in their frequency content (Fig. 3). In addition, bursts are coherent with the low-frequency content of the stimulus. For these reasons, we propose that bursts are detectors of low-frequency stimulus features. The situation for isolated spikes is more complex (see below).

Because the optimal features detected by bursts and isolated spikes during $0-60 \mathrm{~Hz}$ stimulation differ, we cannot directly assess the discrimination performance of bursts or single spikes 
when they are coding similar frequency ranges. Thus, we directly compared the feature detection capability of bursts and isolated spikes for narrow bands of LF $(0-20 \mathrm{~Hz})$ and $\mathrm{HF}(40-60 \mathrm{~Hz})$ stimuli. In response to LF stimuli, bursts have better SNRs than isolated spikes $\left(\mathrm{SNR}_{\mathrm{B}}, 3.76 \pm\right.$ $0.23 ; \mathrm{SNR}_{\mathrm{I}}, 2.36 \pm 0.26 ; p<0.05$ ) (Fig. $7 \mathrm{C1}$, inset). The optimal feature detected by bursts is a scaled version of the isolated spike feature with the burst feature having a higher amplitude threshold for bursting (Fig. 7C2). Thus, bursts are more selective for their stimulus features than single spikes. Conversely, the lower threshold for isolated spikes leads to tonic firing that tracks the stimulus whenever the membrane potential is above threshold. This improves coherence and therefore stimulus estimation (Fig. 2) but reduces the difference between the optimal feature and the null feature, which hinders feature detection.

Alternatively, when presented an HF stimulus $(40-60 \mathrm{~Hz})$, isolated spikes have high SNR values (Fig. 7D1, inset). Although the lack of bursts prevents a statistical comparison between isolated spikes and bursts, in four of six cells, the SNR for isolated spikes was greater than for the full spike train, suggesting that rare instances of bursting may actually compromise high-frequency stimulus detection.

These results demonstrate that bursts, although not necessary for stimulus estimation, offer a significant advantage over isolated spikes for low frequency feature detection. Conversely, isolated spikes are better estimators of low-frequency stimuli. At high frequencies, isolated spikes could be considered as either feature detectors or stimulus estimators depending on the downstream decoding mechanism.

\section{Burst dynamics are required for feature detection}

The implication that bursts are more reliable feature detectors than isolated spikes is based on the hypothesis that sequential activation at a synapse by two or more spikes separated by short ISIs is more effective than when the spikes are separated by long ISIs (Lisman, 1997). This raises the question of whether short ISIs that are a consequence of biophysical burst mechanism provide an advantage for information transfer as compared with short ISIs that are not the result of a burst mechanism. We again used the ghostburster model to investigate whether bursting dynamics are required to generate high-frequency multispike events that are selective for low frequencies. In Figure 8, three scenarios are presented in which we attempted to recreate the low-frequency selectivity and the feature extraction in the model neuron with a PDM to match that of the ADM. As shown previously, removing burst dynamics shifted the ISI histogram from bimodal (Fig. $8 A 1$ ) to unimodal (Fig. 8A2-A4) distributions. Because HF events are no longer well defined, we identified subsets of $\mathrm{HF}$ events on the basis of ISI criteria (Fig. $8 A 2-A 4$, colored vertical lines) that resulted in the same $\mathrm{HF}$ event rate $(5 \mathrm{~Hz})$ or fraction (0.24) as indicated by the bimodal histogram of the ADM. In the first scenario, we changed the definition of an HF event from an ISI $<6 \mathrm{msec}$ to an ISI $<10 \mathrm{msec}$, such that the mean firing rate and the HF event rate and fraction were the same as the ADM. The HF events were low pass (Fig. 8 B; ADM, black; PDM $10 \mathrm{msec}$ ISI, blue) but were poor feature detectors compared with true ADM bursts (Fig. 8C; control, black; $10 \mathrm{msec}$ ISI, blue).

To ensure that reduced feature detection in the PDM was not attributable to the loss of ISIs $<6 \mathrm{msec}$, in the second and third scenarios we increased the offset current, $I_{0}$, injected into the PDM and used $6 \mathrm{msec}$ criterion to distinguish HF events (Fig. $8 A 3, A 4)$. In the second scenario, the fraction of HF events with ISIs $<6$ msec was comparable with the burst fraction in the ADM. The PDM required 18 times more current to generate the same fraction of ISIs $<6 \mathrm{msec}$ as the ADM, which increased the mean firing rate to $80 \mathrm{~Hz}$ and the $\mathrm{HF}$ event rate to $15 \mathrm{~Hz}$. This resulted in broadband HF event coherence (Borst and Haag, 2001) but very poor feature detection (Fig. $8 B, C$, red lines).

In the final scenario, the rate of ISI events $<6 \mathrm{msec}$ was com- 

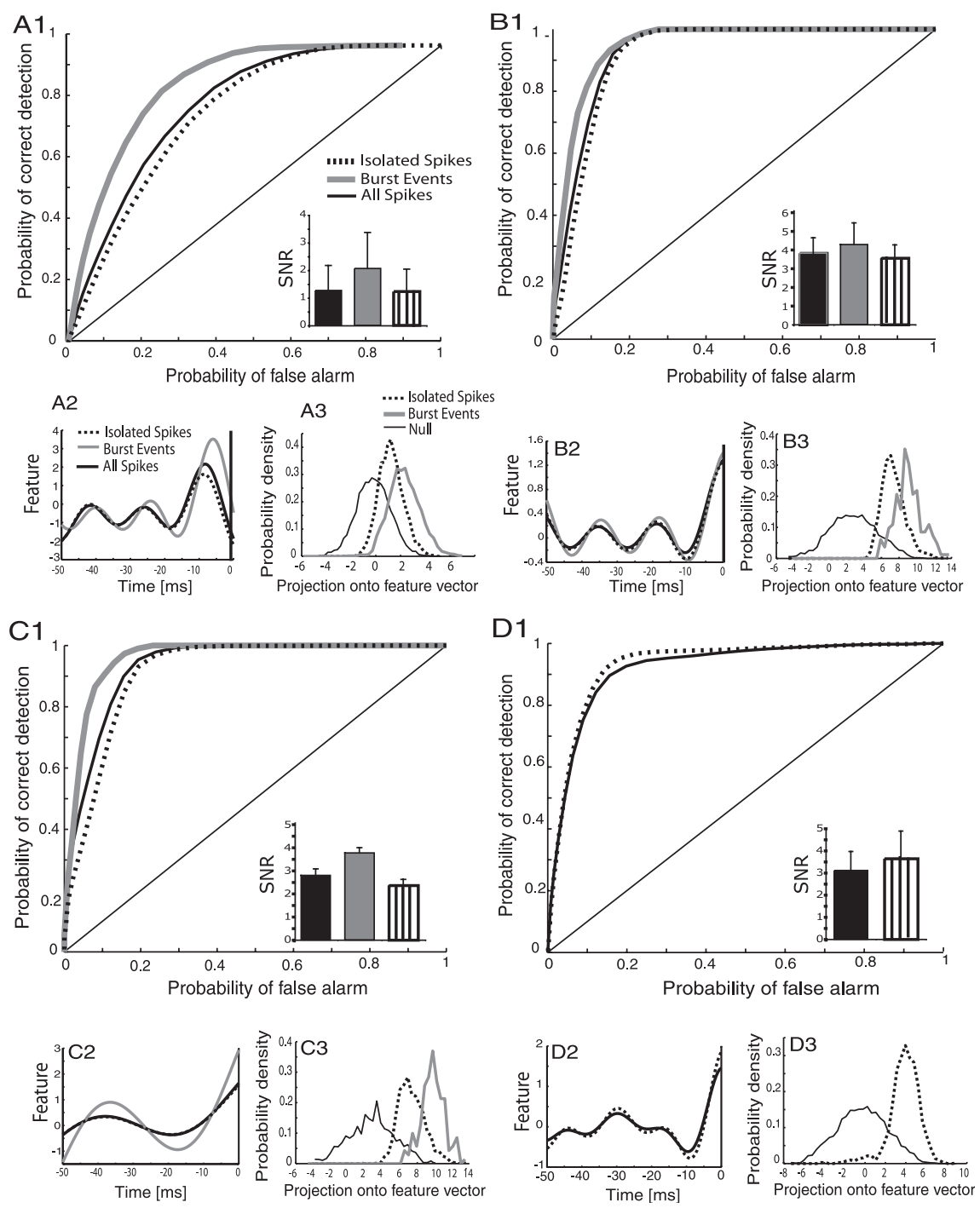

Figure 7. Feature extraction in vivo and in vitro. $A$, Analysis of spike trains in response to a broadband stimulus in vivo. $B$, Analysis of spike trains in response to a broadband stimulus in vitro. C, Analysis of spike trains in response to LF stimulation (0-20 $\mathrm{Hz}$ ) in vitro. D, Analysis of spike trains in response to $\mathrm{HF}$ stimulation $(40-60 \mathrm{~Hz})$ in vitro. A1-D1, ROC curves for the partitioned spike trains: all spikes (black solid line), bursts (thick gray line), and isolated spikes (black dashed line). Inset, The mean SNR of all spikes (black solid line), bursts (gray line), and isolated spikes (black striped line). A2-D2, The optimal features detected by all spikes (black solid line), bursts (gray line), and isolated spikes (black dashed line). Note that extended structure of these features (less than $-20 \mathrm{msec}$ ) may be attributable to the narrow-band nature of the stimulus set. This may induce long-range correlations in the stimulus waveforms that might not be causally related to spikes and not eliminated by spike-triggered averaging. A3-D3, Projection onto the optimal features, bursts (gray line), isolated spikes (black dashed line), and the null feature (black thin line).

tribution (Fig. 8E) leading to a higher probability of false alarm. The end result is a reduced ability to detect specific stimulus features, even when the high-frequency event rate is conserved. Thus, backpropagation-dependent pyramidal cell bursts are not only low-frequency selective, but the burst mechanism enables pyramidal cells to generate a distinct stream of high-frequency burst events that demonstrate superior feature extraction capability over nonburst HF events.

\section{Discussion}

We demonstrated both in vivo and in vitro that in response to broadband stimuli, ELL pyramidal cells generate a spike train that consists of two parallel information streams. Specifically, ELL pyramidal cells selectively detect low-frequency features of broadband Gaussian stimuli with spike bursts, whereas single spikes simultaneously estimate the entire stimulus frequency range.

Interestingly, the low-frequency coherence of pyramidal cell responses was not dependent on burst dynamics, because the coherence between full spike train and the stimulus was unaffected by the dendritic application of TTX in vitro and by removing dendritic $\mathrm{Na}^{+}$conductance in the ghostburster model. Under these conditions, a low-frequency event triggers a somatic spike, which is coherent with the stimulus. However, this spike fails to backpropagate, and additional burst spikes do not occur. This demonstrates that the lowfrequency coherence with the stimulus is carried in the timing of the first spike of the burst (Kepecs and Lisman, 2003). Because coherence is directly related to the linear estimation of a signal from a spike train (Gabbiani and Koch, 1998; Roddey et al., 2000), we conclude that bursts are not necessary for this estimate.

We quantified the selectivity of bursting neurons using a feature extraction technique described previously (Gabbiani

parable with the burst rate in the ADM. Again, to produce an $\mathrm{HF}$ event rate of $5 \mathrm{~Hz}$, the passive neuron required five times the current, resulting in a higher mean firing rate $(50 \mathrm{~Hz})$ than the ADM. These events were low-frequency coherent but also failed to recover the same degree of feature extraction as true bursts (Fig. $8 B, C$, green lines).

These results demonstrate that neurons with passive dendrites can generate $\mathrm{HF}$ events (ISIs $<6$ or $<10 \mathrm{msec}$ ) that are coherent with low frequencies. However, in each instance, the optimal feature detected by the HF event is of lower amplitude at the time of the event $(t=0)$ compared with the ADM (Fig. $8 D$, black). This decreases the distinction between the distribution of features detected by HF events and the null distribution. When stimulus features are projected onto these optimal features, there is less distinction between the HF event distributions and the null dis- et al., 1996; Metzner et al., 1998). Although single spikes are sufficient to estimate low-frequency input, our in vitro results demonstrate that bursts are substantially better detectors of these signals. Although this corroborates previous in vivo results (Gabbiani et al., 1996; Metzner et al., 1998), we expand on these studies by showing that feature detection by bursts is low-frequency specific and, at least in some cells, dependent on the backpropagation-dependent burst mechanism.

\section{Benefits of burst dynamics in neural coding}

The analysis of feature extraction by the model neuron with intact burst dynamics revealed that bursts were superior feature detectors even when compared with similar high-frequency spike events produced by the model neuron with a passive dendrite. Moreover, the passive dendrite model required substantially 
higher input current than the bursting model neuron to generate comparable numbers of high-frequency spike sequences, which significantly increased the mean firing rate. Thus, whereas coherence and stimulus estimation are enhanced by increases in firing rate (Borst and Haag, 2001), the reduced feature selectivity of the nonbursting neuron shows that these high-frequency events cannot adequately substitute for bursts when they are used as feature detectors.

Furthermore, it has been argued that neural codes should be sparse and implement low firing rates to be efficient (Rolls and Tovee, 1995; Vinje and Gallant, 2000). Burst dynamics may allow for such codes, because they permit low firing rates while having a significant proportion of distinct high-frequency burst events. We therefore propose that pyramidal cells may use highfrequency bursts to selectively detect and efficiently code for the low-frequency components of stimuli.

\section{Decoding pyramidal cell output}

For bursts to be an important component of a neural code, an appropriate postsynaptic decoding mechanism is required. For instance, a presynaptic burst ISI may match a resonance timescale of a postsynaptic neuron and gate information transfer (Izhikevich et al., 2003). Alternatively, bursts in conjunction with short-term synaptic plasticity could improve information transfer at unreliable synapses (Lisman, 1997). ELL pyramidal cell output is decoded in the torus semicircularis (TS) of the midbrain, and both synaptic depression and facilitation may contribute to the selective extraction of low-frequency features. Fortune and Rose (1997) demonstrated that different TS cells in a related electric fish are tuned for various stimulus-frequency ranges (low pass, broadband, and high pass). The output of the TS is conveyed to brain regions selectively responsible for electrolocation (low frequency, optic tectum) or electrocommunication (low to high frequencies, nucleus electrosensorius) (Bastian, 1982; Heiligenberg et al., 1991; Bell and Maler, 2004). A number of TS neurons temporally filters electrosensory stimuli resulting in low-pass $(3-8 \mathrm{~Hz})$, broadband $(0-30 \mathrm{~Hz})$, and high-pass cells $(>10 \mathrm{~Hz})$ (Fortune and Rose, 1997). In the case of low-pass cells, responses to stimulus frequencies $>10 \mathrm{~Hz}$ are attenuated by synaptic depression. Interestingly, this depression can be overcome because these cells also show marked facilitation to high frequency ( $\sim 10 \mathrm{msec}$ ISI) presynaptic activity (Fortune and Rose, 2001). In this study, we have shown that ELL pyramidal cells convert low-frequency sensory input to high-frequency bursts. Thus, these bursts provide the high-frequency events that can be reliably detected by TS low-pass neurons. ELL pyramidal cells can therefore simultaneously code for both low-frequency stimulus components via bursts as well as high frequency components via isolated spikes. Different TS cell populations (low pass, broad-
A2 $\quad B$
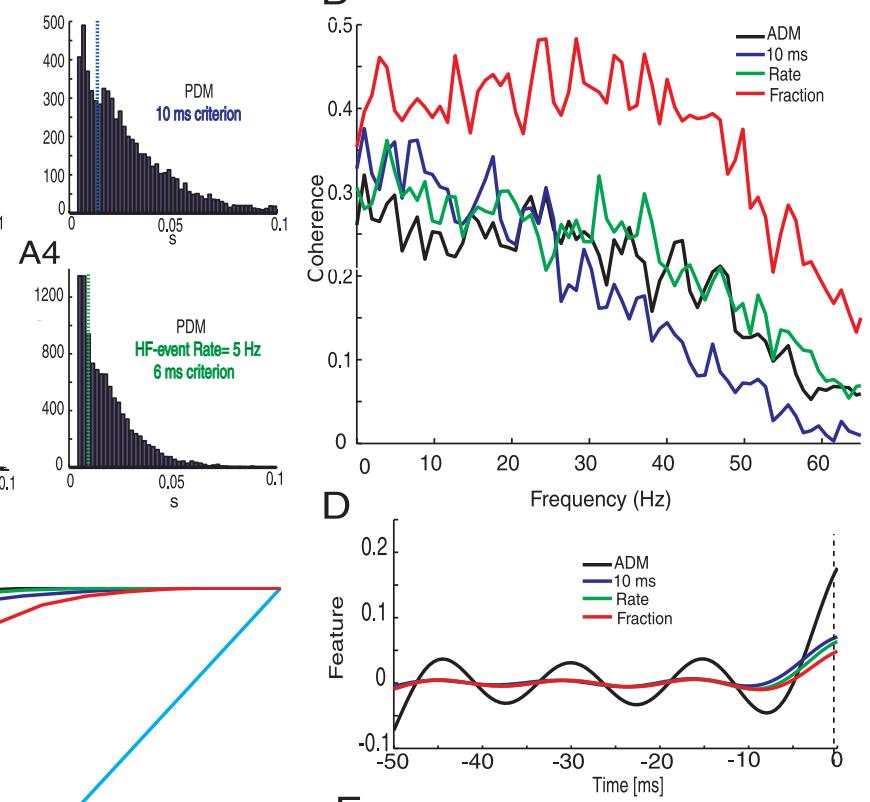

E

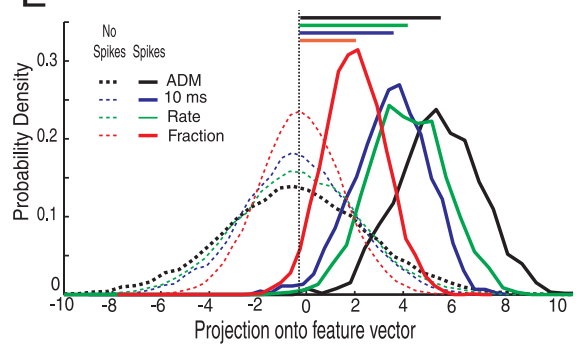

Figure 8. Burst dynamics are required for feature detection. $A$, ISI histograms in response to broadband stimulation of the bursts (see Materials and Methods); firing rate, $33 \mathrm{~Hz}$. A2, Unimodal histogram of the PDM; blue line indicates $10 \mathrm{msec}$ ISI criterion ( 24 ) and rate $(5 \mathrm{~Hz}$ ) of $\mathrm{HF}$ events seen in the ADM; firing rate, $33 \mathrm{~Hz}$. A3, PDM histogram in response (red line). A4, PDM histogram in response to an increase in $I_{0}$ that increased the firing rate to $50 \mathrm{~Hz}$ and conserved the rate of $<6$ (colors as determined and indicated in $A$ ). C, ROC curves of bursts (ADM; black) or HF events (PDM; colors as in A). D, Optima features detected bursts or HF events in each scenario. $E$, Feature projections in each scenario onto the optimal feature (solid lines) as compared with the null feature (dashed lines).

band, or high pass) can then selectively decode information related to low-frequency signals emanating from prey versus highfrequency communication signals.

\section{The ELL and the thalamus as sensory gateways}

The parallels between the ELL-toral connections and thalamocortical pathways have been reviewed recently (Berman and Maler, 1999; Krahe and Gabbiani, 2004). Thalamic bursting has been both biophysically characterized and modeled (Lu et al., 1992; McCormick and Huguenard, 1992; Mukherjee and Kaplan, 1995; Smith et al., 2000). In lateral geniculate (LGN) relay cells, bursts have been shown to exhibit frequency tuning (Mukherjee and Kaplan, 1995; Smith et al., 2000; Fortune and Rose 2001) and enhance detectability at stimulus onset (Sherman, 2001; Martinez-Conde et al., 2002). Furthermore, it has been suggested that depending on the specific LGN-cortical layer projection, bursts and single spikes could present distinct streams of information transfer for the same stimulus features (Reinagel et al., 1999) and that feedback from the cortex could switch coding 
modes between signal detection and stimulus estimation (Sherman, 2001).

Likewise, ELL pyramidal cell bursting is regulated through several dendritic and somatic ionic conductances (Noonan et al., 2003) and could conceivably be modulated by feedback input to the apical dendrites (Berman and Maler, 1999; Bastian and Nguyenkim, 2001). Recent studies have shown that ELL pyramidal cell responses to sensory input depend on both the frequency content and spatial extent of the stimulus (Bastian et al., 2002; Chacron et al., 2003; Doiron et al., 2003a) and that feedback is differentially activated by spatially localized versus spatially extended stimuli (Doiron et al., 2003a; Bastian et al., 2004). Thus, not only could feedback to the ELL switch coding strategies from feature detection by bursts to stimulus estimation by single spikes as proposed for the thalamus, but it could also lead to the differential coding of distinct behaviorally relevant low-frequency (prey like) versus high-frequency (communication) stimuli.

\section{References}

Bastian J (1982) Vision and electroreception: integration of sensory information in the optic tectum of the weakly electric fish Apteronotus albifrons. J Comp Physiol 147:287-297.

Bastian J (1996a) Plasticity in an electrosensory system. I. General features of a dynamic sensory filter. J Neurophysiol 76:2483-2496.

Bastian J (1996b) Plasticity in an electrosensory system. II. Postsynaptic events associated with a dynamic sensory filter. J Neurophysiol 76:2497-2507.

Bastian J, Nguyenkim J (2001) Dendritic modulation of burst-like firing in sensory neurons. J Neurophysiol 85:10-22.

Bastian J, Chacron MJ, Maler L (2002) Receptive field organization determines pyramidal cell stimulus-encoding capability and spatial stimulus selectivity. J Neurosci 22:4577-4590.

Bastian J, Chacron MJ, Maler L (2004) Plastic and non plastic cells perform unique roles in a network capable of adaptive redundancy reduction. Neuron 41:767-779.

Bell CC, Maler L (2004) Central neuroanatomy of electrosensory systems in fish. In: Evolution of the vertebrate auditory system, Vol 22 (Manley GA, Popper AN, Fay RR, eds). Berlin: Springer.

Berman NJ, Maler L (1998) Inhibition evoked from primary afferents in the electrosensory lateral line lobe of the weakly electric fish. J Neurophysiol 80:3173-3196.

Berman NJ, Maler L (1999) Neural architecture of the electrosensory lateral line lobe: adaptations for coincidence detection, a sensory searchlight and frequency-dependent adaptive filtering. J Exp Biol 202:1243-1253.

Berman NJ, Plant J, Turner R, Maler L (1997) Excitatory amino acid transmission at a feedback pathway in the electrosensory system. J Neurophysiol 78:1869-1881.

Borst A, Haag J (2001) Effects of mean firing on neural information rate. J Comput Neurosci 10:213-221.

Chacron MJ, Doiron B, Maler L, Longtin A, Bastian J (2003) Non-classical receptive field mediates switch in a sensory neuron's frequency tuning. Nature 423:77-81.

Debusk BC, Debruyn EJ, Snider RK, Kabara JF, Bonds AB (1997) Stimulusdependent modulation of spike burst length in cat striate cortical cells. J Neurophysiol 78:199-213.

Doiron B, Longtin A, Turner RW, Maler L (2001) Model of gamma frequency burst discharge generated by conditional backpropagation. J Neurophysiol 86:1523-1545.

Doiron B, Laing C, Longtin A, Maler L (2002) Ghostbursting: a novel neuronal burst mechanism. J Comput Neurosci 12:5-25.

Doiron B, Chacron M, Maler L, Longtin A, Bastian J (2003a) Inhibitory feedback required for network oscillatory responses but not prey stimuli. Nature 421:539-543.

Doiron B, Noonan L, Lemon N, Turner RW (2003b) Persistent Na + current modifies burst discharge by regulating conditional backpropagation of dendritic spikes. J Neurophysiol 89:324-337.
Eggermont JJ (1998) Is there a neural code? Neurosci Biobehav Rev 22:355-370.

Fortune ES, Rose GJ (1997) Passive and active membrane properties contribute to the temporal filtering properties of midbrain neurons in vivo. J Neurosci 17:3815-3825.

Fortune ES, Rose GJ (2001) Short-term synaptic plasticity as a temporal filter. Trends Neurosci 24:381-385.

Gabbiani F, Koch C (1998) Principles of spike train analysis. In: Methods in neuronal modeling (Koch C, Segev I, eds), pp 313-360. Cambridge, MA: MIT.

Gabbiani F, Metzner W, Wessel R, Koch C (1996) From stimulus encoding to feature extraction in weakly electric fish. Nature 384:564-567.

Heiligenberg W, Keller CH, Metzner W, Kawasaki M (1991) Structure and function of neurons in the complex of the nucleus electrosensorius of the gymnotiform fish Eigenmannia: detection and processing of electric signals in social communication. J Comp Physiol A 169:151-164.

Herrington J, Newton KR, Bookman RJ (1995) B PULSE CONTROL V4.5: IGOR XOPS for patch clamp data acquisition and capacitance measurements. Miami, FL.

Izhikevich EM, Desai NS, Walcott EC, Hoppensteadt FC (2003) Bursts as a unit of neural information: selective communication via resonance. Trends Neurosci 26:161-167.

Keat J, Reinagel P, Reid RC, Meister M (2001) Predicting every spike: a model for the responses of visual neurons. Neuron 30:803-817.

Kepecs A, Lisman J (2003) Information encoding and computation with spikes and bursts. Network 14:103-118.

Kepecs A, Wang X-J, Lisman J (2002) Bursting neurons signal input slope. J Neurosci 22:9053-9062.

Koch C (1999) Biophysics of computation: information processing in single neurons. New York: Oxford UP.

Koch C, Segev I (1999) The Hodgkin-Huxley model of action potential generation. In: Biophysics of computation (Stryker M, ed). New York: Oxford UP.

Krahe R, Gabbiani F (2004) Burst firing in sensory systems. Nat Neurosci 5:13-23.

Laing CR, Longtin A (2003) Periodic forcing of a model sensory neuron. Phys Rev E Stat Nonlin Soft Matter Phys 67:051928.

Laing CR, Doiron B, Longtin A, Noonan L, Turner RW, Maler L (2003) Type 1 burst excitability. J Comput Neurosci 14:329-342.

Lemon N, Turner RW (2000) Conditional spike backpropagation generates burst discharge in a sensory neuron. J Neurophysiol 84:1519-1530.

Lestienne R (2001) Spike timing, synchronization and information processing on the sensory side of the central nervous system. Prog Neurobiol 65:545-591.

Lisman J (1997) Bursts as a unit of neural information: making unreliable synapses reliable. Trends Neurosci 20:38-43.

Lu SM, Guido W, Sherman SM (1992) Effects of membrane voltage on receptive field properties of lateral geniculate neurons in the cat: contribution of the low-threshold $\mathrm{Ca}^{2+}$ conductance. J Neurophysiol 68:2185-2198.

MacIver MA, Sharabash NM, Nelson ME (2001) Prey capture behavior in gymnotid electric fish: motion analysis and effects of water conductivity. J Exp Biol 204:543-557.

Malinow R, Otmakhov N, Blum KI, Lisman J (1994) Visualizing hippocampal synaptic function by optical detection of $\mathrm{Ca} 2+$ entry through the N-methyl-D-aspartate channel. Proc Natl Acad Sci USA 91:8170-8174.

Martinez-Conde S, Macknik S, Hubel D (2002) The function of bursts of spikes during visual fixation in the awake primate lateral geniculate nucleus and primary visual cortex. Proc Natl Acad Sci USA 99:13920-13925.

McCormick DA, Huguenard JR (1992) A model of the electrophysiological properties of thalamocortical relay neurons. J Neurophysiol 68:1384-1400.

Metzner W, Koch C, Wessel R, Gabbiani F (1998) Feature extraction by burstlike spike patterns in multiple sensory maps. J Neurosci 18:2283-2300.

Mukherjee P, Kaplan E (1995) Dynamics of neurons in the cat lateral geniculate nucleus: in vivo electrophysiology and computational modeling. J Neurophysiol 74:1222-1243.

Noonan L, Doiron B, Laing C, Longtin A, Turner RW (2003) A dynamic dendritic refractory period regulates burst discharge in the electrosensory lobe of weakly electric fish. J Neurosci 23:1524-1534. 
Reinagel P, Godwin D, Sherman M, Koch C (1999) Encoding of visual information by LGN bursts. J Neurophysiol 81:2558-2569.

Rieke F, Warland D, de Ruyter van Steveninck RR, Bialek W (1997) Spikes: exploring the neural code. Cambridge, MA: MIT.

Roddey JC, Girish B, Miller JP (2000) Assessing the performance of neural encoding models in the presence of noise. J Comput Neurosci 8:95-112.

Rolls ET, Tovee MJ (1995) Sparseness of the neuronal representation of stimuli in the primate temporal visual cortex. J Neurophysiol 73:713-726.

Sherman SM (2001) Tonic and burst firing: dual modes of thalamocortical relay. Trends Neurosci 24:122-126.

Smith GD, Cox CL, Sherman M, Rinzel J (2000) Fourier analysis of sinusoi- dally driven thalamocortical relay neurons and a minimal integrate-andfire-or-burst model. J Neurophysiol 83:588-610.

Turner RW, Maler L, Deerinick T, Levinson SR, Ellisman MH (1994) TTXsensitive dendritic sodium channels underlie oscillatory discharge in a vertebrate sensory neuron. J Neurosci 14:6453-6471.

Turner RW, Plant JR, Maler L (1996) Oscillatory and burst discharge across electrosensory topographic maps. J Neurophysiol 76:2364-2382.

Vinje WE, Gallant JL (2000) Sparse coding and decorrelation in primary visual cortex during natural vision. Science 287:1273-1276.

Zupanc GKH, Maler L (1993) Evoked chirping in the weakly electric fish Apteronotus leptorhynchus: a quantitative biophysical analysis. Can J Zool 71:2301-2310. 